(2) Open Access Full Text Article

\title{
Association of atorvastatin with the risk of hepatotoxicity: a pilot prescription sequence symmetry analysis
}

This article was published in the following Dove Press journal:

Therapeutics and Clinical Risk Management

\section{Haiping Zhang' \\ Jiani Wu' \\ Zhuolin Zhang ${ }^{2}$ \\ Haisheng Qian ${ }^{3}$ \\ Yifan Wang' \\ Miaomiao Yang \\ Yinchu Cheng ${ }^{4}$ \\ Shaowen Tang'}

'Department of Epidemiology, School of Public Health, Nanjing Medical University, Nanjing 2III66, Jiangsu, People's Republic of China; ${ }^{2}$ Department of Clinical Pharmacy, School of Pharmacy, Nanjing Medical University, Nanjing 21 I 166, Jiangsu, People's Republic of China; ${ }^{3}$ Department of Internal Medicine, The First Clinical Medical College, Nanjing Medical University, Nanjing, Jiangsu 210029, People's Republic of China; ${ }^{4}$ Department of Pharmacy, Peking University Third Hospital, Beijing 100191, People's Republic of China
Correspondence: Shaowen Tang Department of Epidemiology, School of Public Health, Nanjing Medical University, I0I Longmian Ave, Jiangning District, Nanjing 2III66, People's Republic of China

Tel +862586868224

Fax +862586868499

Email tomswen@njmu.edu.cn
Purpose: This study aimed to evaluate Atorvastatin (ATO)-associated hepatotoxicity using prescription sequence symmetry analysis (PSSA), based on a health insurance database of a Chinese population living in Jiangsu Province, China.

Methods: Patients prescribed ATO and hepatoprotective drugs in 2017 were identified, and the run-in period was determined based on the "waiting-time" distribution. Adjusted sequence ratio (ASR) and 95\% confidence interval $(95 \% \mathrm{CI})$ were calculated to estimate the risk of ATO-associated hepatotoxicity under different time intervals or based on gender and age stratification.

Results: A total of 2,549 patients, with 1,518 filling the ATO prescription first and 1,031 filling the ATO prescription second, were analyzed. After setting the run-in period as 30 days and the time interval as $15,30,60,90,120$, and 180 days, the ASRs were 1.492 (95\% CI: 1.367-1.652), 1.399 (95\% CI: 1.308-1.508), 1.280 (95\% CI: 1.213-1.357), 1.292 (95\% CI: 1.234-1.356), 1.278 (95\% CI: 1.226-1.336), and 1.274 (95\% CI: 1.229-1.323), respectively. No significant difference was observed between different genders and ages $\left(\chi^{2}=0.161\right.$, $P=0.688 ; \chi^{2}=1.565, P=0.211$, respectively).

Conclusion: This is the first study conducted in a real-world setting to evaluate the relationship between ATO and hepatotoxicity using the PSSA in a Chinese population. We found a 1.3- to 1.5-fold increase in risk of hepatotoxicity following ATO, with the greater risk occurring within the first 30 days of treatment.

Keywords: atorvastatin, hepatoprotective drug, prescription sequence symmetry analysis, health insurance database

\section{Introduction}

Atorvastatin (ATO) is one of the most widely prescribed drugs and statins in the world; ${ }^{1}$ it decreases production of low-density lipoprotein (LDL) cholesterol by blocking the action of the 3-hydroxy-3-methylglutaryl-coenzyme A (HMG-CoA) reductase enzyme in the liver at the rate-limiting step of cholesterol biosynthesis. ${ }^{2}$ Together with other statins, ATO has been commonly used for decades. Currently, ATO is proven to play other roles in decreasing the risk of heart failure, ${ }^{3}$ cerebrovascular disease, ${ }^{4}$ chronic subdural hematoma, ${ }^{5}$ depression, ${ }^{6}$ pancreatic cancer, ${ }^{7}$ contrast-induced acute kidney injury, ${ }^{8}$ rheumatoid arthritis, ${ }^{9}$ and so on. Although ATO is safe and generally well tolerated across the range of its therapeutic dosage $(10-80 \mathrm{mg} /$ day $) ;^{10}$ it is associated with adverse effects, which are underrecognized as well as underreported. ${ }^{11,12}$ Common adverse drug events (ADEs) or adverse drug 
reactions (ADRs) for patients taking ATO include arthralgia, dyspepsia, diarrhea, nausea, nasopharyngitis, insomnia, urinary tract infection and pain in the extremities. ${ }^{13}$ Additionally, ATO-associated myalgia, ${ }^{11}$ hearing loss, ${ }^{14}$ and hepatotoxicity ${ }^{15,16}$ are often reported. Idiosyncratic liver injury associated with statins is rare but can be associated with severe outcomes, ${ }^{17}$ and the research on this topic is incomplete but extremely necessary. ${ }^{18}$

Postmarketing surveillance is essential in order to protect patients against avoidable risks from medication. ${ }^{19}$ Spontaneous reporting on ADRs is a quick method, but underreporting is a problem; intensive monitoring gives high quality data but is expensive. ${ }^{19}$ Now, there is ongoing interest in developing systems or methods that can incorporate and use existing electronic data to enable active surveillance for $\mathrm{ADEs},{ }^{20}$ and this surveillance for signal detection of ADEs is feasible. ${ }^{21}$ Prescription sequence symmetry analysis (PSSA) is a signal detection method for ADEs utilizing administrative claims data ${ }^{22}$ and is based on analyzing the sequences of medications; if one medication (marker drug) is more often initiated after another medication (index drug) than before, it may be an indication of an adverse effect of the index drug. ${ }^{23}$ Validation studies have indicated that the PSSA has moderate sensitivity and high specificity, and has robust performance. ${ }^{23}$ PSSA has become a tool to assist in global pharmacosurveillance of medicines, complementary to other methods, and a pharmacovigilance tool to identify unsuspected side effects. $^{23,24}$ The method has been increasingly used to investigate safety concerns of medications, including ace-inhibitor induced cough, inhaled corticosteroid induced oral candidiasis, nonsteroidal anti-inflammatory induced stroke, and isotretinoin and cardiovascular medicine induced depression. ${ }^{25}$ To our knowledge, only one Chinese study reported the association between statins and liver injury using PSSA, ${ }^{26}$ but no other study has evaluated ATO-associated hepatotoxicity based on the PSSA.

The health insurance data are an important source of information for medical research, ${ }^{27}$ which usually contains each patient's unique encrypted identification number, age, sex, diagnosis, medical treatment administered, whether the individual was an inpatient or outpatient, type of insurance, medical expenses, medical institution identification number, and prescriptions. The data are a valuable resource for exploratory analyses of a variety of health services research questions. ${ }^{27}$ Based on the PSSA, the health insurance database can also be used for the study of ADEs/ADRs. This study aimed to evaluate ATOassociated hepatotoxicity using the PSSA, based on a health insurance database of a Chinese population in Jiangsu Province, China.

\section{Patients and methods}

\section{Data source}

Data for this study were obtained from the health insurance database of Jiangsu Province in 2017. A subset of data was used in the present study, including patients who were prescribed ATO or hepatoprotective drugs as single or combination products in either the inpatient or the outpatient setting. The variables in the data set included drug prescriptions (generic name and brand name of drugs, and prescription time) and patients' information (identification number, gender, age). The present study was approved by the Ethics Committee of Nanjing Medical University, and the data set does not contain any information that can identify subjects, such as names or addresses.

\section{Definition of index and marker drugs}

The index drug is the drug thought to produce a given side effect, while the marker drug is the drug used to alleviate the given side effect. ${ }^{28}$ In the present study, the index drug is ATO, regardless of its manufacturer or dosage. The marker drugs, namely, hepatoprotective drugs, which were the most commonly used drugs in China, were categorized into five therapeutic classes: anti-inflammatory, antioxidant, antidote, choleretic, cell membrane repair agents (Table 1). Such categorization applied regardless of the manufacturer or dosage. In China, the patients with

Table I Hepatoprotective drugs included in the atorvastatin analyses

\begin{tabular}{|l|l|}
\hline Therapeutic class & Hepatoprotective drugs \\
\hline Anti-inflammatory agents & Magnesium Isoglycyrrhizinate, Diammonium glycyrrhizinate \\
Antioxidant agents & Bicyclol, Bifendate \\
Antidote agents & Reduced glutathione sodium for injection \\
Choleretic agents & Ademetionine I,4-Buta nedisulfonate, Ursodeoxycholic acid \\
Cell membrane repair agent & Polyene phosphatidylcholine \\
\hline
\end{tabular}


drug-induced liver injury (DILI) can be treated with hepatoprotective drugs. ${ }^{29}$ The international criteria established by the Council for International Organizations of Medical Sciences (CIOMS) is used for judging the DILI based on three clinical types. ${ }^{29,30}$ (1) hepatocellular injury, alanine aminotransferase (ALT) $\geq 3$ upper limit of normal (ULN) and $\mathrm{R}$ (the ratio of the elevation of baseline ALT to baseline alkaline phosphatase (ALP)) $\geq 5$; (2) cholestatic injury, ALP $\geq 2$ ULN and $\mathrm{R} \leq 2$; (3) hepatocellular-cholestatic mixed injury, ALT $\geq 3$ ULN, ALP $\geq 2$ ULN and $2<\mathrm{R}<5$.

\section{Run-in period and time periods}

The run-in period, namely, how long you would have to wait before a patient fills his first prescription for a given drug, was determined based on the "waiting-time" distribution. ${ }^{22,31}$ The time intervals, the periods between the initiation of index drugs and marker drugs, were set at different days $(15,30,60,90,120$, and 180 days) to conduct the sensitivity analysis according to the reference. ${ }^{26}$

\section{Statistical analysis}

The PSSA method, originally described by Hallas, ${ }^{22}$ was applied in this study. In brief, PSSA proceeds in three steps. First, patients who filled incident prescriptions of both index and marker drugs during 2017 are identified. Second, patients are classified on the basis of the temporal order of the alternative sequences of prescription events. The "causal" group consists of patients who filled the index drug first and the marker drug second, whereas conversely the "noncausal" group consists of patients who filled the index drug second and the marker drug first. Finally, the estimate of risk is calculated by taking the ratio of the number of patients in the "causal" to the "noncausal" groups. ${ }^{22,32}$ The three key indicators were calculated as follows:

(1) The crude sequence ratio (CSR) is the ratio of the number of patients in the "causal" group over the number of patients in the "noncausal" group, which is a measure of the degree of asymmetry between the two patient groups.

(2) The null-effect sequence ratio (NESR) determines the expected sequence ratio given no cause-effect relationship between the index drug and the marker drug. The NESR measures differential change in prescribing patterns during the study period, which could confound the CSR. NESR is defined as $a /(1-a)^{33}$ where $a$ is given as

$$
a=\frac{\sum_{m=1}^{u} A T O_{m} \sum_{n=m+1}^{m+d} H D_{n}}{\sum_{m=1}^{u} A T O_{m}\left(\sum_{n=m-d}^{m-1} H D_{n}+\sum_{n=m+1}^{m+d} H D_{n}\right)}
$$

In the aforementioned formula, $m$ or $n$ is the consecutive days of the survey period (excluding the run-in period) and $u$ is the last day of the survey period. HD is an abbreviation for hepatoprotective drug. $\mathrm{HD}_{\text {index }}$ is the number of patients being prescribed hepatoprotective drugs first on the index day, and $\mathrm{ATO}_{\text {index }}$ is the number of patients being prescribed ATO first on the index day. $d$ is the time interval between ATO and the hepatoprotective drugs.

(3) The adjusted sequence ratio (ASR) was calculated for each time interval as the CSR divided by the NESR $(A S R=C S R / N E S R) .95 \%$ confidence intervals $(95 \% \mathrm{CI})$ for the ASRs were calculated by using a method for exact confidence intervals in binomial distributions. ${ }^{34}$

Stratified analysis was conducted according to different genders and ages, and the difference between the two groups was analyzed by the $\chi^{2}$ test. Findings were considered statistically significant at an alpha level of 0.05 if the 95\% CI did not include the null (1.0). All calculations were performed using STATA (version 13.0, Stata Corp, College Station, Texas, USA).

\section{Results}

The study database contains records on 10,318 patients with 73,388 prescriptions of ATO or hepatoprotective drugs over the period from January 1, 2017, to December 31, 2017. Among these patients, 4,480 patients prescribed both ATO and hepatoprotective drugs in the same year were identified. Five hundred eighty-two patients were excluded from the analysis because of being prescribed both drugs on the same day. As shown in Figure 1, the "waiting-time" distribution curves showed a steeply descending limb, reaching a more or less stable plateau after 30 days. Therefore, the other 1,349 patients were excluded because the run-in period was less than 30 days. Of the remaining 2,549 patients, 1,518 filled the ATO prescription first and 1,031 filled the ATO prescription second (Figure 2). A slight asymmetry was observed in the distribution between prescription orders (Figure 3).

After setting the time interval between the initiation of index and marker drugs as 15, 30, 60, 90, 120, and 180 days, the ASRs were 1.492 (95\% CI: 1.367-1.652), 


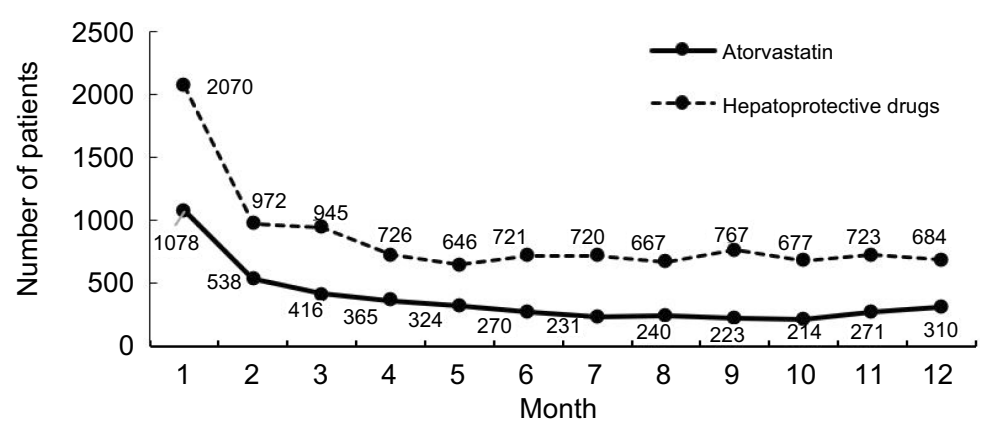

Figure I Waiting-time distributions for patients of atorvastatin and hepatoprotective drugs during the period January 2017 to December 2017.

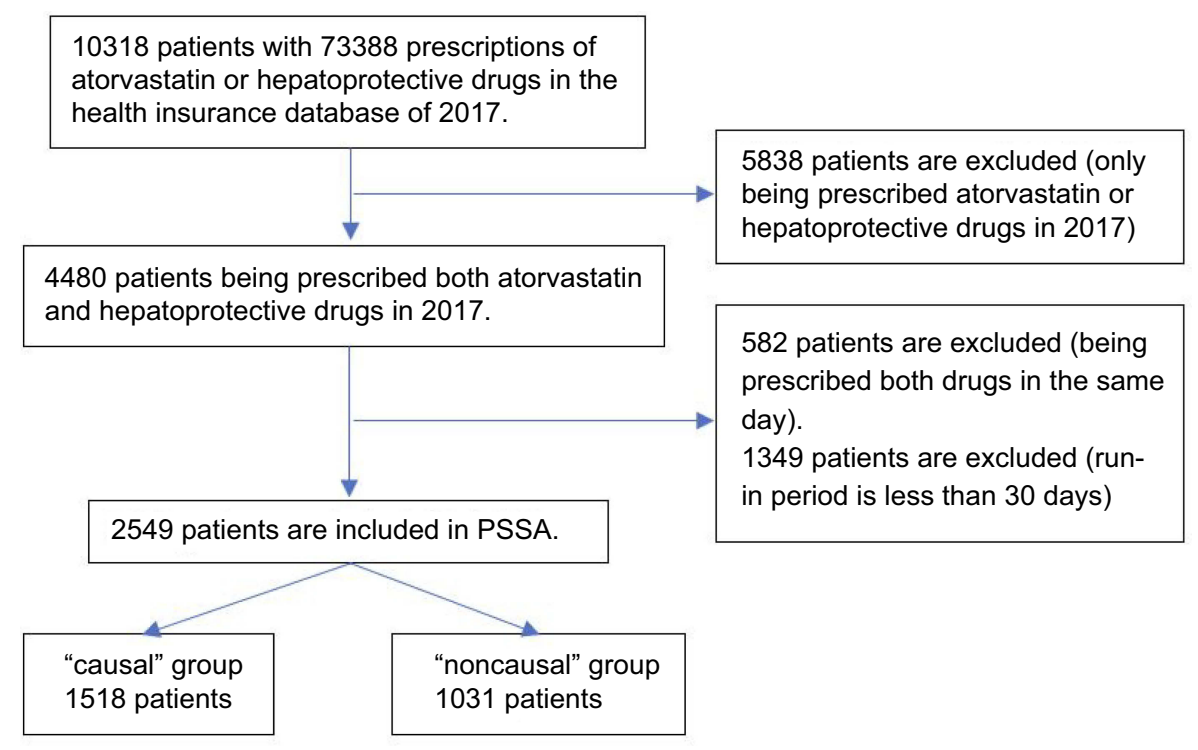

Figure 2 A flow chart of patients' inclusion and exclusion.

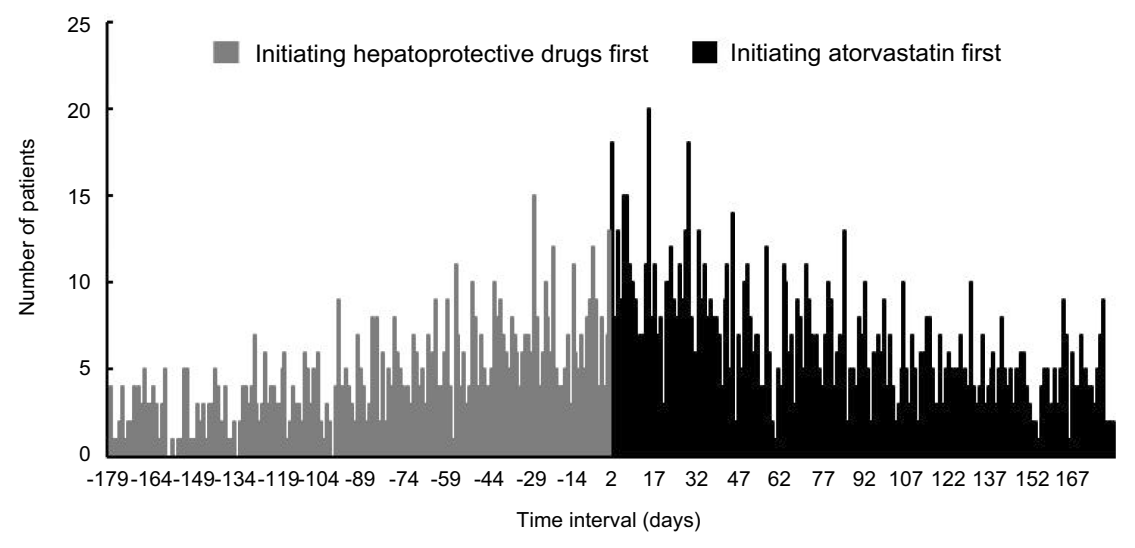

Figure 3 Frequency distribution of patients by days before or after atorvastatin initiation within 180 days.

$1.399 \quad(95 \% \quad$ CI: $\quad 1.308-1.508), \quad 1.280 \quad(95 \% \quad$ CI: $\quad$ trend as the extension of the time interval, and the positive $1.213-1.357), 1.292$ (95\% CI: $1.234-1.356), 1.278(95 \%$ signal was stronger within 30 days' time interval.

CI: $1.226-1.336)$, and 1.274 (95\% CI: $1.229-1.323)$,

An extended stratified analysis was conducted accordrespectively (Table 2). The ASRs showed a downward ing to different genders and ages, and the results are shown 


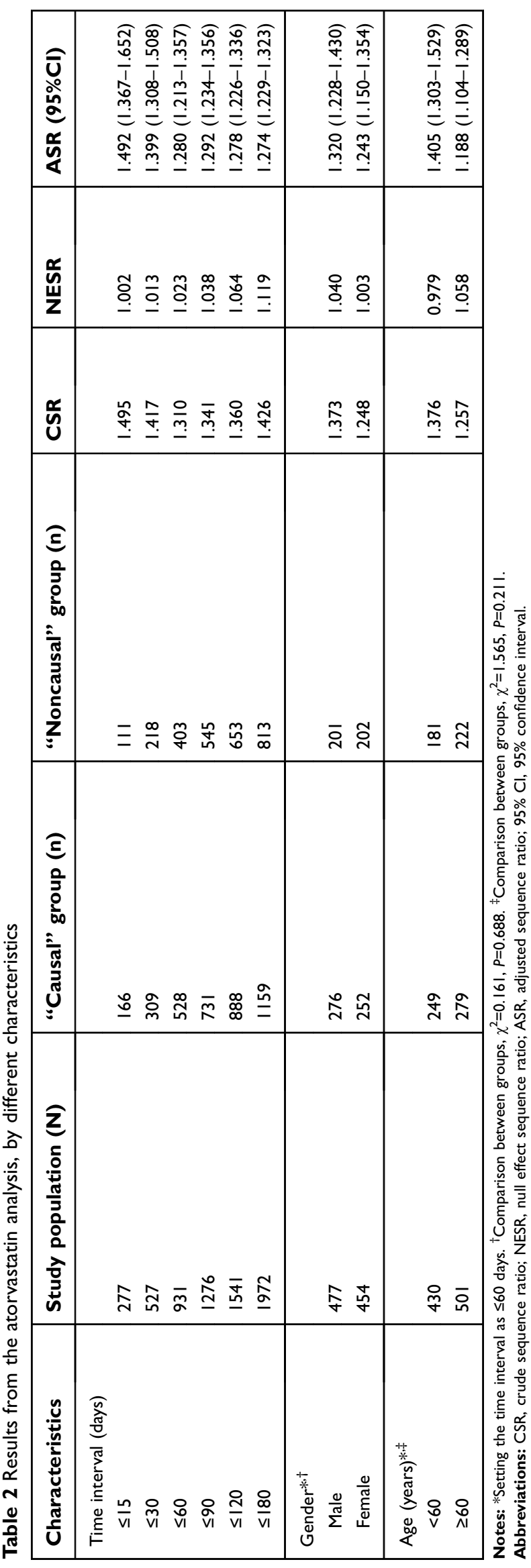

in Table 2. The ASRs were 1.320 (95\% CI: 1.228-1.430) and 1.243 (95\% CI: $1.150-1.354)$ in males and females, respectively; 1.405 (95\% CI: $1.303-1.529)$ and 1.188 (95\% CI: 1.104-1.289) in patients age $<60$ and $\geq 60$ years, respectively. No significant difference was observed between different genders and ages $\left(\chi^{2}=0.161\right.$, $P=0.688 ; \chi^{2}=1.565, P=0.211$, respectively).

\section{Discussion}

This study is the first to evaluate the association of ATO with the potential risk of hepatotoxicity using PSSA in a Chinese population. Based on the health insurance database and the PSSA, we confirmed the relationship between the index drug (ATO) and the marker drugs (hepatoprotective drugs) (The ASRs were more than 1 under different time intervals, and 95\% CI did not include the null), which indicated that the possibility of ATO-induced hepatotoxicity in a Chinese population. There is a $1.3-$ to 1.5 -fold increase in the risk of hepatotoxicity in the present study, especially within 30 days of initiating ATO first. To date, only one study has explored the relationship between statins and the risk of hepatotoxicity, which showed the potential association between statins and liver injury. ${ }^{26} \mathrm{In}$ that study, the ASR of statins was $1.471 \quad(95 \% \mathrm{CI}$ : 1.395-1.550), and the ASR of ATO was 1.419 (95\% CI: $1.335-1.508),{ }^{26}$ similar to that of the present study. Additionally, the results of ADRs monitoring from different countries also suggested that ATO may cause hepatotoxicity. ADRs reports received by the Swedish Adverse Drug Reactions Advisory Committee in 1988-2010 showed that a statin-related DILI was reported in 1.2/ 100,000 users, and ATO was implicated in 30/73 (41\%) of the cases. ${ }^{17}$ Between April 1994 and August 2012, the Spanish Hepatotoxicity Registry indicated that statinrelated DILI was not common in Spain, but ATO was the statin involved in the greatest number of incidents $(16 / 47$, $34 \%) .{ }^{35}$ In prospective studies of patients with DILI, statins have been the cause in approximately $2-5 \%$ of patients, and ATO has been the most frequently implicated statin in all of the series of statin induced hepatotoxicity. ${ }^{16}$ The present study also verified ATO-associated hepatotoxicity using PSSA, and this signal detection method may be a fast and effective one in drug safety evaluation and can also be implemented based on a health insurance database.

Although rare, ATO-induced hepatotoxicity has been well documented, and different hepatotoxicity mechanisms have been proposed. ${ }^{18}$ Multiple studies suggested that 
ATO-induced autoimmune hepatitis is responsible for most idiosyncratic drug reactions. ${ }^{16,36,37}$ However, no significant association was observed for human leukocyte antigen-A, -B and -C alleles with ATO-induced hepatotoxicity in a Japanese population. ${ }^{38}$ Evidence from cell and animal experiments suggest that it may also be related to other mechanisms. HMG-CoA reductase inhibitors can induce apoptosis by increasing intracellular reactive oxygen species generation. ${ }^{39}$ ATO caused liver tissue dysfunction as well as hepatic cell death via oxidative stress-induced cell signaling pathways, including the signals from the mitochondria, caspases and calpain in a dose-dependent manner. $^{40}$ Thymoquinone, a prominent constituent of Nigella sativa, has antioxidant, anti-inflammatory and antiapoptotic activity, and posttreatment thymoquinone can reverse high-dose ATO-induced hepatic oxidative injury in rats. ${ }^{41}$ Additionally, inhibition of HMG-CoA reductase by statins reduces the production of mevalonate, a precursor of coenzyme Q10. ${ }^{42}$ Even brief exposure to atorvastatin causes a marked decrease in blood coenzyme Q10 concentration. ${ }^{43}$ Coenzyme Q10 is one of the most significant lipid antioxidants and its co-administration has been shown to improve particular atorvastatin side effects stemming from oxidative stress. ${ }^{44,45}$ Therefore, oxidative stress induced by ATO or its metabolites, and the reduction of coenzyme Q10 may contribute to the development of ATO-induced hepatotoxicity. Furthermore, ATO and its metabolites are predominantly eliminated by ATPbinding cassette (ABC) transporters (ABCB1 and ABCG2) mediated transport from liver into bile. ${ }^{46}$ A variation (rs2032582) in $A B C B 1$ was also significantly associated with ATO-induced hepatotoxicity ( $\mathrm{G}$ allele versus $\mathrm{T}$ and $\mathrm{A}$ alleles, $\mathrm{OR}=2.59,95 \% \mathrm{CI}$ : $1.49-4.50$, $P=0.00068) .{ }^{38}$ All these potential mechanisms, on the one hand, illustrate the complexity of the mechanism of ATO-induced hepatotoxicity, and on the other hand, indicate that ATO does cause liver injury in patients initiating ATO. Therefore, it is necessary to strengthen patient monitoring and timely detect cases of hepatotoxicity, especially within 30 days of initiating ATO.

In addition to ATO, other statins have been implicated in the liver injury as well. ${ }^{18}$ However, only ATO and simvastatin have been associated with fatality from statin induced liver injury. ${ }^{16}$ Therefore, if the patients cannot tolerate ATO, other statins could be used for lipidlowering treatments, such as fluvastatin, lovastatin, pravastatin and rosuvastatin. ${ }^{16}$ In addition, some natural lipid-lowering drugs are also safe, effective and well tolerated in Chinese patients, such as Chinese medicine Zhabitai, $^{47}$ Xuezhikang ${ }^{48}$ and so on.

\section{Limitations and strengths}

Our study has several strengths. PSSA is a simple form of a self-controlled design that is able to analyze effects of drugs normally used for chronic conditions, and the effect of the measured and unmeasured confounders is automatically canceled out when the effect is stable over the study period. ${ }^{33}$ Although this is a pilot study based on data for one year, we only included all first-time patients of ATO and hepatoprotective drugs to exclude the influence of nonfirst-time patients. The run-in period was determined by plotting the number of patients who were first-time users each month to find the stable plateau. Sensitivity analysis under different time intervals was used to detect the signal and find which time period the signal was stronger. Our study also has some limitations. First, using hepatoprotective drugs as a proxy for hepatotoxicity is not perfect. On the one hand, it is possible that some physicians would discontinue ATO treatment if they suspected that abnormal liver function in patients was related to ATO. On the other hand, in addition to the hepatoprotective drugs mentioned in this study, there are some other Chinese herbal medicines that may be used to treat hepatotoxicity but were not included as the marker drugs. Second, we did not investigate the dose-response relationship between ATO and hepatotoxicity in the study because only patients who had both the marker and index drugs were included in the analyses. Third, although generic ATO is as effective as the brand-name drug in lowering cholesterol levels, ${ }^{49}$ we didn't distinguish whether ATO was a generic or brand name drug because of the limited sample size.

\section{Conclusion}

To the best of our knowledge, this is the first study conducted in a real-world setting to evaluate the relationship between ATO and hepatotoxicity using the PSSA in a Chinese population. We found a 1.3- to 1.5-fold increase in risk of hepatotoxicity following ATO, with the greater risk occurring within the first 30 days of treatment. Our findings may help guide patient education at the initiation of treatment with ATO for lowering cholesterol levels. PSSA is effectively applied as a safety signal detection tool for recently marketed medicines, and would be more widely used in the monitoring of ADEs/ADRs signals in future. 


\section{Acknowledgments}

This study was funded by the National Natural Science Foundation of China (81373066) and Priority Academic Program Development of Jiangsu Higher Education Institutions (PAPD).

\section{Author contributions}

Haiping Zhang, Miaomiao Yang, Yinchu Cheng, and Shaowen Tang contributed to the study concept and design and take responsibility for the integrity of the data and the accuracy of the data analysis. Haiping Zhang, Jiani $\mathrm{Wu}$, Zhuolin Zhang, Haisheng Qian, Yifan Wang and Miaomiao Yang obtained and conducted statistical analysis. Haiping Zhang drafted the report, which was edited by Shaowen Tang. All authors contributed toward data analysis, drafting and critically revising the paper and agree to be accountable for all aspects of the work. All authors have reviewed and approved the final version of manuscript.

\section{Disclosure}

The authors report no conflicts of interest in this work.

\section{References}

1. Adams SP, Tsang M, Wright JM. Lipid-lowering efficacy of atorvastatin. Cochrane Database Syst Rev. 2015;12(3):Cd008226.

2. Burnett JR, Wilcox LJ, Telford DE, et al. Inhibition of HMG-CoA reductase by atorvastatin decreases both VLDL and LDL apolipoprotein B production in miniature pigs. Arterioscler Thromb Vasc Biol. 1997;17(11):2589-2600.

3. Duan HY, Liu DM, Qian P, et al. Effect of atorvastatin on plasma NT-proBNP and inflammatory cytokine expression in patients with heart failure. Genet Mol Res. 2015;14(4):15739-15748. doi:10.4238/ 2015.December.1.25

4. Zhong P, Wu D, Ye X, et al. Secondary prevention of major cerebrovascular events with seven different statins: a multi-treatment meta-analysis. Drug Des Devel Ther. 2017;11:2517-2526. doi:10.2147/DDDT.S135785

5. Qiu S, Zhuo W, Sun C, Su Z, Yan A, Shen L. Effects of atorvastatin on chronic subdural hematoma: a systematic review. Medicine. 2017;96 (26):e7290. doi:10.1097/MD.0000000000007290

6. Salagre E, Fernandes BS, Dodd S, Brownstein DJ, Berk M. Statins for the treatment of depression: a meta-analysis of randomized, double-blind, placebo-controlled trials. $J$ Affect Disord. 2016;200:235-242. doi:10.1016/j.jad.2016.04.047

7. Archibugi L, Arcidiacono PG, Capurso G. Statin use is associated to a reduced risk of pancreatic cancer: a meta-analysis. Dig Liver Dis. 2019;51(1):28-37. doi: 10.1016/j.dld.2018.09.007

8. Liu LY, Liu Y, Wu MY, Sun YY, Ma FZ. Efficacy of atorvastatin on the prevention of contrast-induced acute kidney injury: a meta-analysis. Drug Des Devel Ther. 2018;12:437-444. doi:10.2147/ DDDT.S149106

9. Li GM, Zhao J, Li B, et al. The anti-inflammatory effects of statins on patients with rheumatoid arthritis: a systemic review and meta-analysis of 15 randomized controlled trials. Autoimmun Rev. 2018;17 (3):215-225. doi:10.1016/j.autrev.2017.10.013
10. Athyros VG, Tziomalos K, Karagiannis A, Mikhailidis DP. Atorvastatin: safety and tolerability. Expert Opin Drug Saf. 2010;9 (4):667-674. doi:10.1517/14740338.2010.495385

11. Indumathi C, Anusha N, Vinod KV, Santhosh S, Dkhar SA. Atorvastatin induced adverse drug reactions among South Indian Tamils. J Clin Diagn Res. 2017;11(7):Fc01-fc05. doi:10.7860/ JCDR/2017/27223.10175

12. Rosenbaum D, Dallongeville J, Sabouret P, Bruckert E. Discontinuation of statin therapy due to muscular side effects: a survey in real life. Nutr Metab Cardiovasc Dis. 2013;23 (9):871-875. doi:10.1016/j.numecd.2012.04.012

13. McIver LA, Siddique MS. Atorvastatin. [Updated January 5, 2019]. In: StatPearls [Internet]. Treasure Island (FL): StatPearls Publishing; 2019 Jan. Available from: https://www.ncbi.nlm.nih.gov/books/ NBK430779/. Accessed June 6, 2019.

14. Liu M, Alafris A, Longo AJ, Cohen H. Irreversible atorvastatin-associated hearing loss. Pharmacotherapy. 2012;32(2): e27-e34. doi:10.1002/PHAR.1040

15. Clarke AT, Johnson PC, Hall GC, Ford I, Mills PR. High dose atorvastatin associated with increased risk of significant hepatotoxicity in comparison to simvastatin in UK GPRD cohort. PLoS One. 2016;11(3):e0151587. doi:10.1371/journal.pone.0151587

16. Bjornsson ES. Hepatotoxicity of statins and other lipid-lowering agents. Liver Int. 2017;37(2):173-178. doi:10.1111/liv.13308

17. Bjornsson E, Jacobsen EI, Kalaitzakis E. Hepatotoxicity associated with statins: reports of idiosyncratic liver injury post-marketing. J Hepatol. 2012;56(2):374-380. doi:10.1016/j.jhep.2011.07.023

18. Karahalil B, Hare E, Koc G, Uslu I, Senturk K, Ozkan Y. Hepatotoxicity associated with statins. Arh Hig Rada Toksikol. 2017;68(4):254-260. doi:10.1515/aiht-2017-68-2994

19. Westerholm B. The rationale for a post-marketing surveillance. Hum Reprod. 1987;2(1):41-44. doi:10.1093/oxfordjournals.humrep. a136486

20. Huang YL, Moon J, Segal JB. A comparison of active adverse event surveillance systems worldwide. Drug Saf. 2014;37(8):581-596. doi:10.1007/s40264-014-0194-3

21. Coloma PM, Trifiro G, Schuemie MJ, et al. Electronic healthcare databases for active drug safety surveillance: is there enough leverage? Pharmacoepidemiol Drug Saf. 2012;21(6):611-621. doi: $10.1002 /$ pds. 3197

22. Hallas J. Evidence of depression provoked by cardiovascular medication: a prescription sequence symmetry analysis. Epidemiology. 1996;7(5):478-484.

23. Lai EC, Pratt N, Hsieh CY, et al. Sequence symmetry analysis in pharmacovigilance and pharmacoepidemiologic studies. Eur J Epidemiol. 2017;32(7):567-582. doi:10.1007/s10654-017-0281-8

24. Pratt N, Chan EW, Choi NK, et al. Prescription sequence symmetry analysis: assessing risk, temporality, and consistency for adverse drug reactions across datasets in five countries. Pharmacoepidemiol Drug Saf. 2015;24(8):858-864. doi:10.1002/pds.3780

25. Pratt NL, Ilomaki J, Raymond C, Roughead EE. The performance of sequence symmetry analysis as a tool for post-market surveillance of newly marketed medicines: a simulation study. BMC Med Res Methodol. 2014;14:66. doi:10.1186/1471-2288-14-66

26. Fang RF, Li JH, Zhang J, Gao SQ, Pan YT, Zhan SY. [Association between statins use and liver injury based on prescription sequence symmetry analysis]. Zhonghua Liu Xing Bing Xue Za Zhi. 2016;37(7):935-939. doi:10.3760/cma.j.issn.02546450.2016.07.006

27. Tyree PT, Lind BK, Lafferty WE. Challenges of using medical insurance claims data for utilization analysis. Am J Med Qual. 2006;21(4):269-275. doi:10.1177/1062860606288774

28. Petri H, Leufkens H, Naus J, Silkens R, van Hessen P, Urquhart J. Rapid method for estimating the risk of acutely controversial side effects of prescription drugs. J Clin Epidemiol. 1990;43 (5):433-439. 
29. Yu YC, Mao YM, Chen CW, et al. CSH guidelines for the diagnosis and treatment of drug-induced liver injury. Hepatol Int. 2017;11 (3):221-241. doi:10.1007/s12072-017-9793-2

30. Chalasani NP, Hayashi PH, Bonkovsky HL, Navarro VJ, Lee WM, Fontana RJ. ACG Clinical Guideline: the diagnosis and management of idiosyncratic drug-induced liver injury. $\mathrm{Am}$ J Gastroenterol. 2014;109(7):950-966; quiz 967. doi:10.1038/ ajg.2014.131

31. Hallas J, Gaist D, Bjerrum L. The waiting time distribution as a graphical approach to epidemiologic measures of drug utilization. Epidemiology. 1997;8(6):666-670.

32. Hersom K, Neary MP, Levaux HP, Klaskala W, Strauss JS. Isotretinoin and antidepressant pharmacotherapy: a prescription sequence symmetry analysis. J Am Acad Dermatol. 2003;49(3):424 432.

33. Kubota K. Symmetry analysis for monitoring safety of newly marketed drugs. Pharmacoepidemiol Drug Saf. 2016;25(3):349-351. doi:10.1002/pds.3886

34. Morris JA, Gardner MJ. Calculating confidence intervals for relative risks (odds ratios) and standardised ratios and rates. BMJ. 1988;296 (6632):1313-1316. doi:10.1136/bmj.296.6632.1313

35. Perdices EV, Medina-Caliz I, Hernando S, et al. Hepatotoxicity associated with statin use: analysis of the cases included in the Spanish Hepatotoxicity Registry. Rev Esp Enferm Dig. 2014;106 (4):246-254

36. Russo MW, Scobey M, Bonkovsky HL. Drug-induced liver injury associated with statins. Semin Liver Dis. 2009;29(4):412-422. doi:10.1055/s-0029-1240010

37. Pelli N, Setti M, Ceppa P, Toncini C, Indiveri F. Autoimmune hepatitis revealed by atorvastatin. Eur J Gastroenterol Hepatol. 2003;15(8):921-924. doi:10.1097/00042737-200308000-00014

38. Fukunaga K, Nakagawa H, Ishikawa T, Kubo M, Mushiroda T. ABCB1 polymorphism is associated with atorvastatin-induced liver injury in Japanese population. BMC Genet. 2016;17(1):79. doi:10.1186/s12863-016-0390-5

39. Qi XF, Zheng L, Lee KJ, et al. HMG-CoA reductase inhibitors induce apoptosis of lymphoma cells by promoting ROS generation and regulating Akt, Erk and p38 signals via suppression of mevalonate pathway. Cell Death Dis. 2013;4:e518. doi:10.1038/ cddis. 2013.44
40. Pal S, Ghosh M, Ghosh S, Bhattacharyya S, Sil PC. Atorvastatin induced hepatic oxidative stress and apoptotic damage via MAPKs, mitochondria, calpain and caspase12 dependent pathways. Food Chem Toxicol. 2015;83:36-47. doi:10.1016/j. fct.2015.05.016

41. Hassan SS, Razzaque A, Ahmad Z, Pazdernik V, Amin SN. Does posttreatment thymoquinone reverse high-dose atorvastatin-induced hepatic oxidative injury in rats? Can J Physiol Pharmacol. 2018;96 (1):51-59. doi:10.1139/cjpp-2017-0599

42. Nawarskas JJ. HMG-CoA reductase inhibitors and coenzyme Q10. Cardiol Rev. 2005;13(2):76-79. doi:10.1097/01.crd.0000154790.42 283.a1

43. Rundek T, Naini A, Sacco R, Coates K, DiMauro S. Atorvastatin decreases the coenzyme Q10 level in the blood of patients at risk for cardiovascular disease and stroke. Arch Neurol. 2004;61(6):889-892. doi:10.1001/archneur.61.6.889

44. Deichmann R, Lavie C, Andrews S. Coenzyme q10 and statin-induced mitochondrial dysfunction. Ochsner J. 2010;10(1):16-21.

45. Pourmoghaddas M, Rabbani M, Shahabi J, Garakyaraghi M, Khanjani R, Hedayat P. Combination of atorvastatin/coenzyme Q10 as adjunctive treatment in congestive heart failure: a double-blind randomized placebo-controlled clinical trial. ARYA Atheroscler. 2014;10(1):1-5.

46. Lee YJ, Lee MG, Lim LA, Jang SB, Chung JY. Effects of SLCO1B1 and $\mathrm{ABCB} 1$ genotypes on the pharmacokinetics of atorvastatin and 2-hydroxyatorvastatin in healthy Korean subjects. Int $J$ Clin Pharmacol Ther. 2010;48(1):36-45.

47. Xu DY, Shu J, Huang QY, et al. Evaluation of the lipid lowering ability, anti-inflammatory effects and clinical safety of intensive therapy with Zhibitai, a Chinese traditional medicine. Atherosclerosis. 2010;211(1):237-241. doi:10.1016/j.atherosclerosis.2010.01.044

48. Moriarty PM, Roth EM, Karns A, et al. Effects of Xuezhikang in patients with dyslipidemia: a multicenter, randomized, placebo-controlled study. J Clin Lipidol. 2014;8(6):568-575. doi:10.1016/j.jacl.2014.09.002

49. Loch A, Bewersdorf JP, Kofink D, Ismail D, Abidin IZ, Veriah RS. Generic atorvastatin is as effective as the brand-name drug (LIPITOR $((\mathrm{R})))$ in lowering cholesterol levels: a cross-sectional retrospective cohort study. BMC Res Notes. 2017;10(1):291. doi:10.1186/s13104-017-2617-6
Therapeutics and Clinical Risk Management

\section{Publish your work in this journal}

Therapeutics and Clinical Risk Management is an international, peerreviewed journal of clinical therapeutics and risk management, focusing on concise rapid reporting of clinical studies in all therapeutic areas, outcomes, safety, and programs for the effective, safe, and sustained use of medicines. This journal is indexed on PubMed Central, CAS,
EMBase, Scopus and the Elsevier Bibliographic databases. The manuscript management system is completely online and includes a very quick and fair peer-review system, which is all easy to use. Visit http://www.dovepress.com/testimonials.php to read real quotes from published authors. 